\title{
Corrugated membranes for improved pattern definition with micro/nanostencil lithography
}

\author{
Marc A.F. van den Boogaart ${ }^{\mathrm{a}, *}$, Maryna Lishchynska ${ }^{\mathrm{b}}$, Lianne M. Doeswijk ${ }^{\mathrm{a}}$, \\ James C. Greer ${ }^{\mathrm{b}}$, Jürgen Brugger ${ }^{\mathrm{a}}$ \\ ${ }^{a}$ Ecole Polytechnique Fédérale de Lausanne (EPFL), Laboratoire de Microsystèmes, CH-1015 Lausanne, Switzerland \\ ${ }^{\mathrm{b}}$ Tyndall National Institute, Lee Maltings, Prospect Row, Cork, Ireland
}

Received 2 June 2005; accepted 30 August 2005

Available online 19 October 2005

\begin{abstract}
We present a MEMS process for the fabrication of arbitrary (adaptable to specific aperture geometries) stabilization of silicon nitride membranes to be used as miniature shadow masks or (nano) stencils. Stabilization was realized by the fabrication of silicon nitride corrugated support structures integrated into large-area thin-film solid-state membranes. These corrugated support structures are aimed to reduce the membrane deformation due to the deposition-induced stress and thus to improve the dimensional control over the surface patterns created by stencil lithography. We have performed physical vapor deposition (PVD) of chromium on unstabilized (standard) stencil membranes and on stabilized (corrugated) stencil membranes to test the proposed stabilization geometry. Both the membrane deformation and the surface structures were analyzed, showing reduced deformation and improved pattern definition for the stabilized stencil membranes. The structures have been modeled using a commercial finite element method (FEM) software tool. The simulation and experimental results confirm that introducing stabilization structures in the membrane can significantly (up to 94\%) reduce out-of-plane deformations of the membrane. The results of this study can be applied as a guideline for the design and fabrication of mechanically stable, complex stencil membranes for direct deposition.
\end{abstract}

(C) 2005 Elsevier B.V. All rights reserved.

Keywords: Shadow mask; Nanostencil; Stress-induced deformation; Mechanical stabilization; Local deposition; FEM modeling

\section{Introduction}

An increased demand for micro- and nanometer scale patterning on "unconventional" surfaces and/or non-IC applications has given rise to new and alternative patterning technologies. These new methods include: indentation of polymers by nanoimprint lithography (NIL) [1,2], local deposition of molecules via a stamp by microcontact printing $(\mu \mathrm{CP})$ or soft-lithography $[3,4]$, or via dip-pen nanolithography (DPN) [5], nanoscale fluidic dispensing (NADIS) [6] and localized material deposition through ultra-miniature shadow masks (nanostencils) [7-10]. The stencil method has the advantage of being a direct vacuum patterning technology, i.e. a controlled amount of material is directly deposited through the stencil apertures without the need for cyclic processing steps used in, for example, resist-based pho-

\footnotetext{
* Corresponding author. Tel.: +41 21 6936741; fax: +41 216936670.

E-mail address: marc.vandenboogaart@epfl.ch (M.A.F. van den Boogaart).
}

tolithography. An additional advantage of stenciling over other patterning methods is its possible non-contact application avoiding cross-contamination or surface damage in case of fragile surfaces, whereas, for example, NIL relies on a hard contact and $\mu \mathrm{CP}$ on a soft contact to transfer the patterns successfully. Recently, we have introduced a process for the fabrication of micro/nanostencils (containing $1 \mathrm{~mm} \times 1 \mathrm{~mm}$ membranes) on a full wafer scale $(100 \mathrm{~mm})$ using a combination of a DUV exposure tool and standard MEMS processing [11]. This type of stencil allows for direct, resistless patterning of mesoscopic surface structures $(200 \mathrm{~nm}$ up to several $100 \mu \mathrm{m})$ at a full wafer scale.

The deposition through stencils suffers, however, from drawbacks such as clogging of apertures and membrane deformation due to the deposition-induced stresses. Clogging occurs when the evaporated material accumulates on top and inside the membrane apertures. This phenomenon changes the shape of the aperture during the deposition process and leads to a distortion of the deposited pattern, eventually resulting in the complete clo- 
(a)

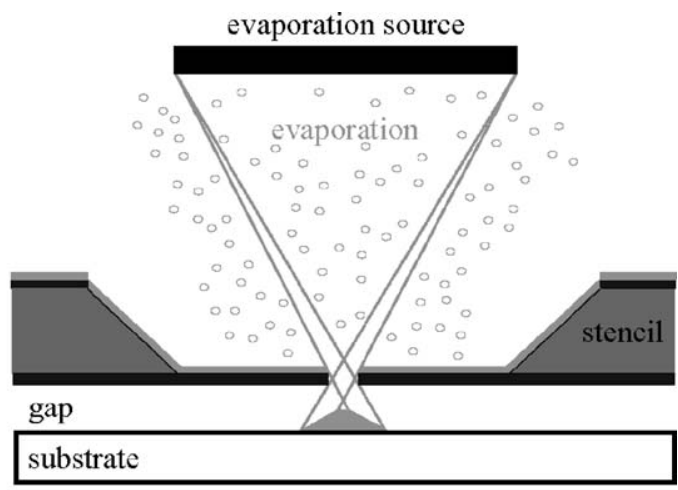

evaporation source

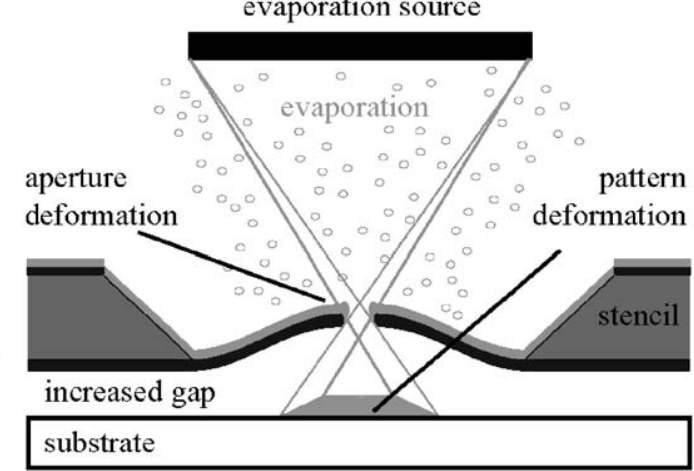

evaporation source

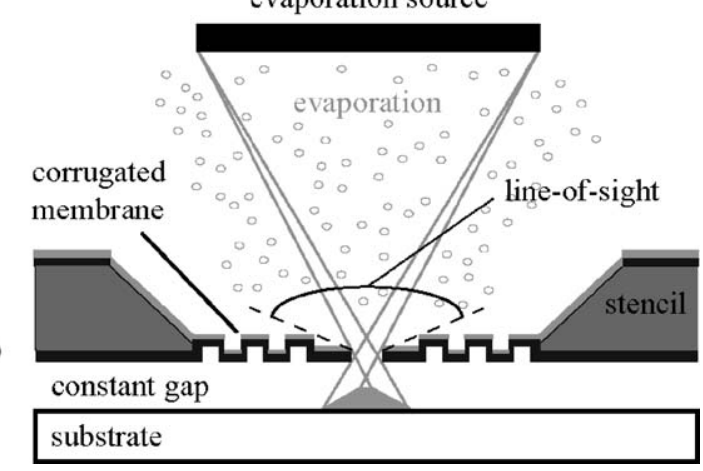

Fig. 1. Schematic representation of a stencil membrane in close proximity of a substrate during evaporation. (a) Ideal stencil deposition. The membrane remains "static" and does not add to pattern deformation. (b) Realistic situation during stencil deposition. The deformed membrane has both an increased gap and aperture deformation resulting in pattern deformation. (c) Deposition through a corrugated stencil. The membrane is locally supported; thus, the membrane deformation and pattern blurring are reduced. sure of the aperture. Membrane deformation results from high levels of in-plane residual stresses caused by the accumulation of the deposited material on the stencil membrane. Since these stencil membranes can contain arbitrary apertures specified by the need for specific surface patterns, the membrane deformation can locally be more severe, resulting in aperture distortion and an increased gap between the substrate and the stencil. This leads to pattern deformation and blurring, i.e. reduced sharpness of edges and limited spatial detail (Fig. 1b).

Residual stresses in MEMS structures arise typically from thin film deposition. These stresses can be of a various nature. When a film on a substrate is subjected to a temperature change during the evaporation, differences in thermal expansion coefficients of the two materials (in this case: film and membrane) result in a thermal stress $\sigma_{\text {th }}$ [12]. All other stresses developed during thin-film deposition fall in the category of intrinsic stress $\sigma_{\text {int }}$. Various physical factors responsible for the intrinsic stress of thin films have been studied $[13,14]$. The difference in thermal expansion coefficients and the lattice spacing mismatch are thought to be the dominant sources of deposition-induced stress causing a substantial deformation of micromachined bi-layered structures.

\section{Design and fabrication of corrugated stencil membranes}

To overcome or minimize the effects of membrane deformation, we have developed a concept in which we mechanically reinforce and thus stabilize the membrane, exploiting an earlier idea presented by Hammer [15]. The improved micro/nanostencils incorporate in situ, local stabilization structures increasing their moment of inertia, $I$, which is the structural property directly related to stiffness or deformability. The stabilization is realized by using a corrugated membrane instead of a planar membrane. Table 1 summarizes cross-sections of three different designs of thin cantilevers (unstabilized, silicon supported and corrugated) with there corresponding $I$ values. It is noteworthy that a single $1-\mu \mathrm{m}$ stabilization rim/corrugation results in a four-fold increase of $I$ with respect to that of the unstabilized (planar) membrane. This increase of $I$ can be achieved with only one additional fabrication step with respect to the stencil fabrication process of planar membranes. The fabrication of Si-supported membranes, however, requires more elaborate processing [16] in order to obtain a similar increase in $I$.

Table 1

Cantilever cross-sections and their corresponding moment of inertia

\begin{tabular}{|c|c|c|c|}
\hline & Unstabilized membrane & Si-supported membrane & Corrugated membrane \\
\hline & & 3 & 3 \\
\hline \multirow[t]{2}{*}{ Cross-section $[\mu \mathrm{m}]$} & n! & 一 & 一 \\
\hline & & 50 & 0 \\
\hline \multirow[t]{4}{*}{ Moment of inertia $\left[\mu \mathrm{m}^{4}\right]$} & 0.52 & 1 rim: 2.88 & 1 rim: 2.05 \\
\hline & & 2 rims: 4.73 & 2 rims: 3.36 \\
\hline & & 3 rims: 6.25 & 3 rims: 4.47 \\
\hline & & 4 rims: 7.53 & 4 rims: 5.41 \\
\hline
\end{tabular}




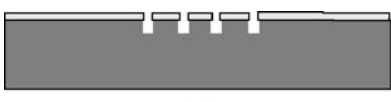

(a)

(b)

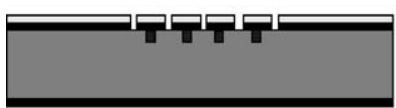

(c)

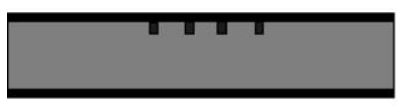

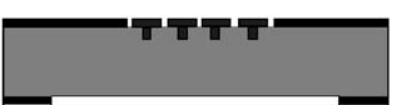

(d)

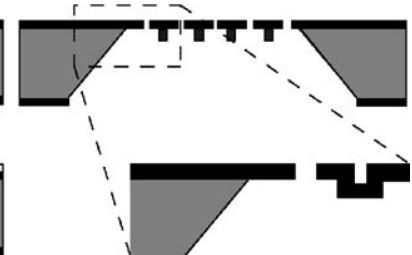

(e)
ऍ $\mathrm{Si}_{\mathrm{SiN}} \rightleftharpoons$ Resist

Fig. 2. Simplified schematic illustration of the fabrication process for a stencil with corrugated membranes: (a) definition of stabilization rims; (b) 500-nm thick LPCVD low-stress SiN deposition; (c) definition of membrane apertures and dry etch transfer of the resist patterns into $\mathrm{SiN}$; (d) membrane etch window definition by backside lithography and pattern transfer; (e) $\mathrm{KOH}$ etching of the bulk Si until membrane is released.

This aperture independent local membrane stabilization is introduced in such a way that it does not interfere with the normal stencil deposition process, i.e. the line of sight of the apertures to the material source is not affected by the stabilization (Fig. 1c). The stabilization allows for the use of larger surface area membranes. We expect to increase the membrane surface area from 1 to $16 \mathrm{~mm}^{2}$ (currently under investigation). In addition, the spacing required between complex membrane apertures to obtain a stable membrane can be reduced by introducing membrane corrugations, increasing the density of complex apertures. Furthermore, it allows for the application of wet cleaning processes due to the increased stability to withstand treatment and handling in fluidic environments, e.g. avoiding the stiction of slender membrane bodies, and thus an increased reusability of the stencils is obtained.

We have fabricated corrugated stencils by means of a silicon micromachining process. Fig. 2 shows a schematic overview of the fabrication process for corrugated stencil membranes. The process begins (Fig. 2a) by defining the stabilization structures or rims in 1.7- $\mu \mathrm{m}$ thick photoresist (S1818, Shipley) by conventional photolithography (MA-150, SUSS MicroTec). The defined structures are subsequently transferred into the topside of the silicon ( $\mathrm{Si}$ ) wafer by inductively coupled plasma (ICP) etching (Alcatel 601E: Bosch process, $\mathrm{SF}_{6} 300 \mathrm{sccm}$ pulsed, $\mathrm{C}_{4} \mathrm{~F}_{8} 150 \mathrm{sccm}$ pulsed, $20^{\circ} \mathrm{C}, 1500 \mathrm{~W}$ ) until a depth of 1,2 or $4 \mu \mathrm{m}$. Then, a 500-nm thick low-stress silicon nitride (LS-SiN)

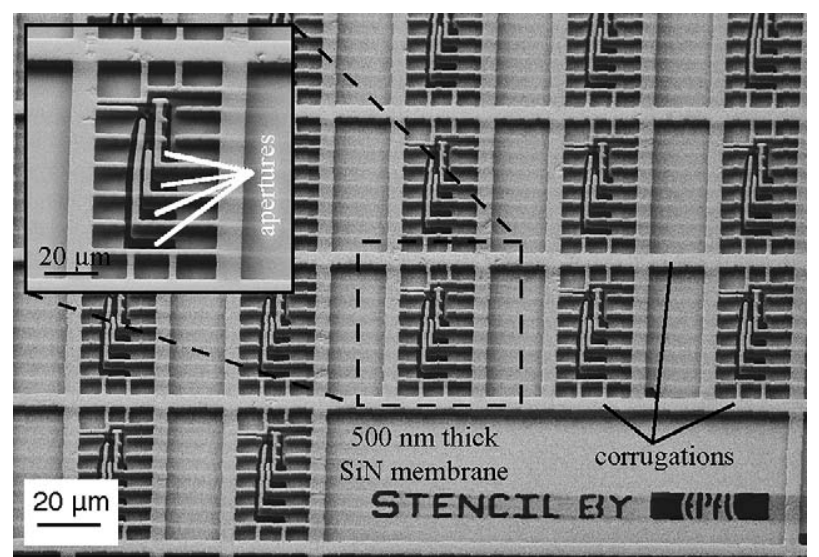

Fig. 4. SEM image of a corrugated membrane for use as a shadow mask.

is deposited (Fig. 2b) by LPCVD (105 sccm $\mathrm{SiH}_{2} \mathrm{Cl}_{2}, 27 \mathrm{sccm}$ $\mathrm{NH}_{3}, 135 \mathrm{mTorr}, 838.5^{\circ} \mathrm{C}$ ). The measured residual stress of the $\mathrm{SiN}$ layer is measured on wafer-scale by wafer curvature measurements (Tencor, FLX-2900) and is approximately $200 \mathrm{MPa}$, tensile. Then, the patterns forming the membrane apertures are defined by optical lithography and transferred into the LSSiN by ICP anisotropic etching (Alcatel 601E: $\mathrm{C}_{2} \mathrm{~F}_{6} 20 \mathrm{sccm}$, $20^{\circ} \mathrm{C}, 1800 \mathrm{~W}$ ) (Fig. 2c). The etch windows for the membrane release are defined by optical lithography and transferred into the backside LS-SiN by ICP anisotropic etching (Alcatel 601E: $\mathrm{C}_{2} \mathrm{~F}_{6} 20 \mathrm{sccm}, 20^{\circ} \mathrm{C}, 1800 \mathrm{~W}$ ). The backside patterned LS-SiN (Fig. 2d) forms the etch mask for the subsequent membrane release by potassium hydroxide etching $\left(\mathrm{KOH}, 40 \mathrm{wt} \%, 60^{\circ} \mathrm{C}\right)$ (Fig. 2e).

Fig. 3 shows the cross-sections of corrugated 500-nm thick membranes with different rim heights after being released from the bulk $\mathrm{Si}$. These cross-sections were made using focused ion beam (FIB) milling (FEI Nova 600 Nanolab, $\mathrm{XeF}_{2}$ gas assisted FIB milling). The cross-sections in Fig. 3 show that the deposited LPCVD SiN membranes have a uniform film thickness and good step coverage of the predefined stabilization structures. Fig. 4 shows a part of a corrugated membrane with apertures meant for the reproduction of nano-electrode circuits currently fabricated using e-beam nanofabrication [17]. The image also demonstrates the possibility of stabilizing arbitrary membrane apertures using various geometries while maintaining a simple stencil fabrication process.
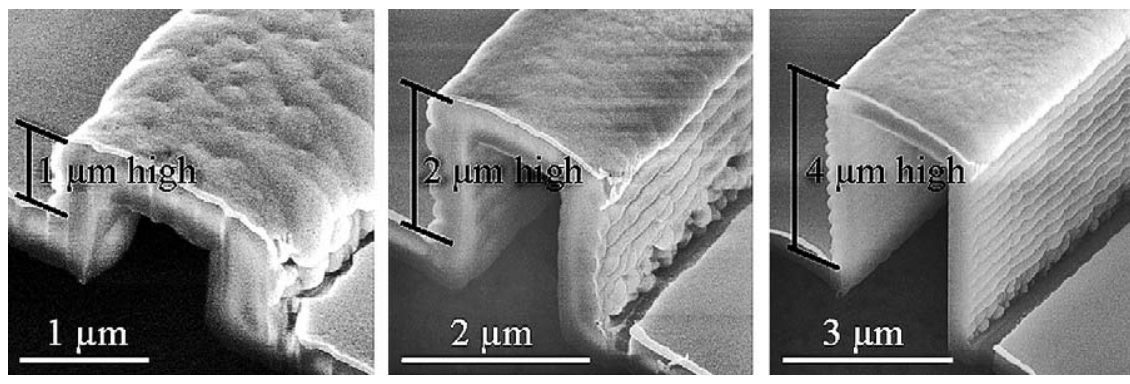

Fig. 3. SEM images of cross-sections of cantilevers with various stabilization heights. 


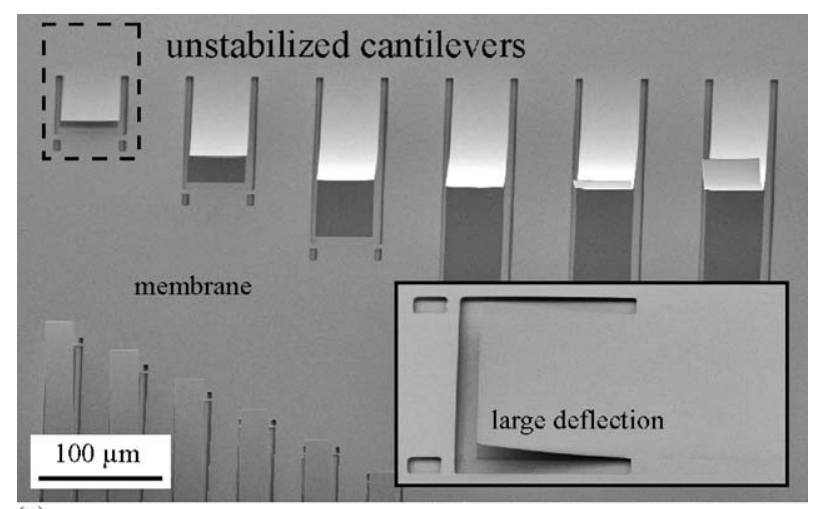

(a)

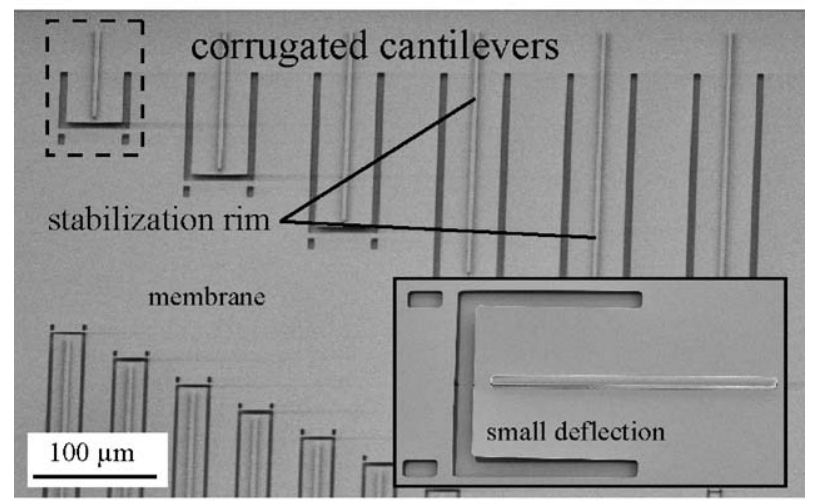

(b)

Fig. 5. SEM image of a membrane after deposition of 50-nm thick Cr: (a) the deposited material induces a significant deflection up to $183 \mu \mathrm{m}$ (for a $300-\mu \mathrm{m}$ long cantilever) for the unstabilized cantilevers and (b) using a 2- $\mu \mathrm{m}$ high single rim reduces the deflection up to $94 \%$ (for a $300-\mu \mathrm{m}$ long cantilever). Both inlets show an $50-\mu \mathrm{m}$ wide and $50-\mu \mathrm{m}$ long cantilever.

\section{Characterization of the improved stencil membranes}

For the experimental verification of the proposed membrane stabilization geometry, we have designed, microfabricated and characterized a series of cantilever-type corrugated membranes with one to four stabilization rims with heights varying from 1 to
$4 \mu \mathrm{m}$. Both stabilized and unstabilized stencil membranes were then coated with a 50-, 70- or 100-nm thick $\mathrm{Cr}$ thin film using electron-beam evaporation (Alcatel EVA $600,4 \times 10^{-7} \mathrm{mbar}$, $0.3 \mathrm{~kW}$, deposition rate: $3-4 \AA / s)$. The stencils were placed perpendicular and centered to the evaporation source at a distance of $50 \mathrm{~cm}$. A supplementary reference wafer was used for determination of the thin-film residual stress by wafer curvature measurements (Tencor, FLX-2900). The deposited Cr film was measured to have a residual stress in the range of $1180-1500 \mathrm{MPa}$ depending on the thickness of the deposited layer.

SEM images of deformed membranes after $\mathrm{Cr}$ deposition for unstabilized and corrugated cantilevers are presented in Fig. 5a and $b$, respectively. A gap of $3 \mu \mathrm{m}$ was present between the membrane and substrate. The deflections of the cantilever-type membranes were determined by measuring the maximum outof-plane deflection of each cantilever with respect to the stable part of the membrane surface (reference plane) by both scanning electron microscopy (SEM, LEO 1550) and an optical surface profiler (Veeco, NT1100). Unstabilized stencil membranes show a large deflection, whereas stabilized stencil membranes demonstrate a significantly reduced deformation. For instance, the end of a 300- $\mu \mathrm{m}$ long unstabilized cantilever has an out-of-plane deflection of $183 \mu \mathrm{m}$. A similar cantilever stabilized by one $1-\mu \mathrm{m}$ high rim has a deflection of only 40 and $10.7 \mu \mathrm{m}$ when reinforced by one $2-\mu \mathrm{m}$ high rim. This measured reduction of the out-of-plane deformation indicates an increased stability of 78 and $94 \%$ for 1 and $2 \mu \mathrm{m}$ high stabilization rims, respectively.

Subsequently, the stencils were removed from the substrate and the surface structures were analyzed by SEM and dynamic force microscopy (DFM). Fig. 6a and b shows images of 45-nm thick $\mathrm{Cr}$ surface structures deposited through both an unstabilized stencil membrane (Fig. 6a) and a corrugated stencil membrane (Fig. 6b). The SEM image and the DFM images in Fig. 6a show a reduced pattern definition and reduced contrast between the deposited $\mathrm{Cr}$ and the Si substrate on the side of maximum membrane deflection. The line scan in Fig. 6a shows the pattern edge definition in more detail. The difference between (a)
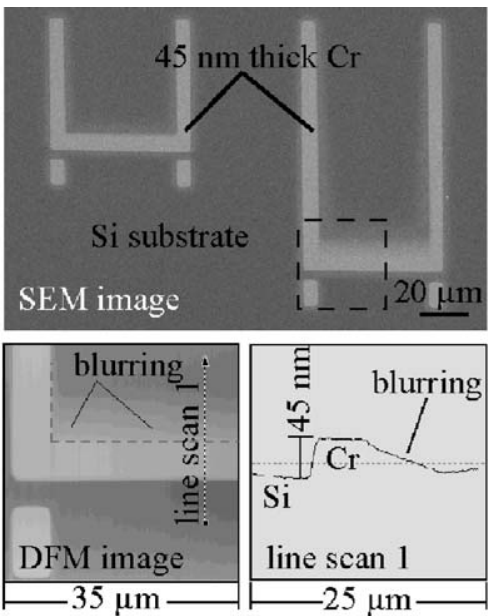

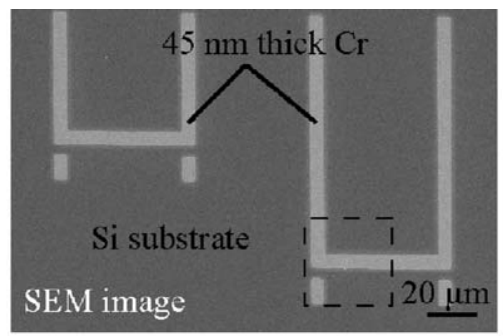

(b)

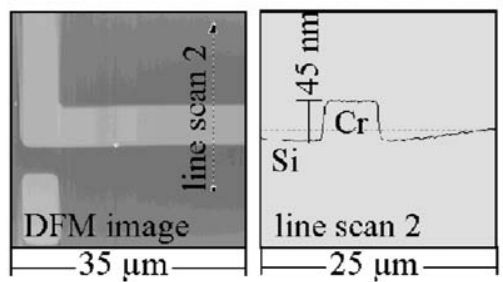

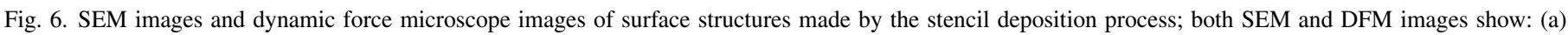

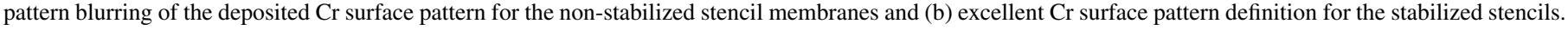


the pattern edge definition on the side of the reference plane and that on the side of the maximum membrane deflection is clearly visible, indicated by a different edge slope (blurring). The SEM image and the DFM images of the surface structures deposited through the stabilized stencil (Fig. 6b) show excellent pattern definition and contrast between the deposited $\mathrm{Cr}$ and Si substrate. The line scan in Fig. 6b shows the symmetrical pattern edge definition on both sides indicating a stable membrane. These results confirm that the corrugated membranes significantly reduce the out-of-plane deformation resulting in an improved surface pattern definition due to a reduced gap increase.

\section{FEM simulation study on membrane deformation and stabilization}

To constructively design the stabilization of complex apertured stencil membranes (as seen in Fig. 4) and develop guidelines for membrane stabilization for future work, we have compared the experimental membrane deformation results with the simulation results of finite elements method (FEM) models. Above-mentioned cantilevers were modeled and simulated with the FEM software tool Coventor [18]. The structures were modeled as bi-layered cantilevers (membrane SiN layer + deposited $\mathrm{Cr}$ layer) and their deformation under the deposition-induced stress was simulated. A truly quantitative theory for determination of deposition-induced stresses is yet to be developed. Therefore, experimental measurements remain the only available method of quantifying the stress and the experimentally measured values of biaxial stress of the $\mathrm{Cr}$ layers were introduced into the simulations. Because the properties of the deposited thin films in this work have not yet been determined, published values for similar deposition processes for the Poisson's ratio and Young's modulus were used for modeling purposes (Table 2).

Comparisons between simulated and measured values of the out-of-plane deflection for different cantilever lengths, deposition thicknesses and stabilization types are shown in Figs. 7-9. The results of the simulation study for the considered geometries of stabilization confirm that a reduction of $80 \%$ in out-of-plane deformation can be achieved by introducing a single stabilization rim of $1 \mu \mathrm{m}$ in height. An increase in the number and height of the rims results in a further reduction (up to $96 \%$ ) in the stress-induced out-of-plane deflection of the cantilever. Both phenomena are best explained by the previously mentioned correlation between the stiffness of the structure and its moment of inertia (Table 1). Thus, introducing more corrugation rims and/or introducing increased rim heights increase the moment of inertia and therefore reduce the membrane deformation. The graphs

Table 2

Material properties used in simulation study

\begin{tabular}{llll}
\hline Material & Young's modulus [GPa] & Poison's ratio & Reference \\
\hline $\mathrm{SiN}$ & 276 & 0.27 & {$[19]$} \\
$\mathrm{Cr}$ & 277 & 0.3 & {$[20]$} \\
\hline
\end{tabular}

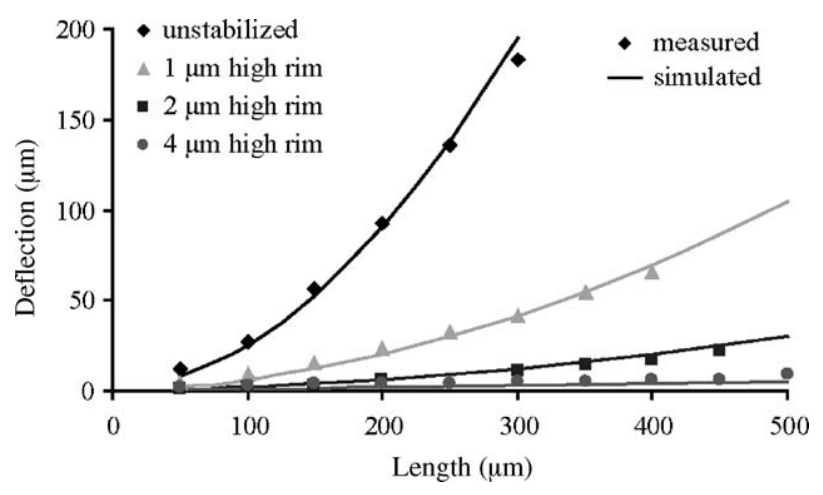

Fig. 7. Plots of simulation results (solid lines) vs. measurement data (dots) for cantilevers stabilized by a single rim of variable heights.

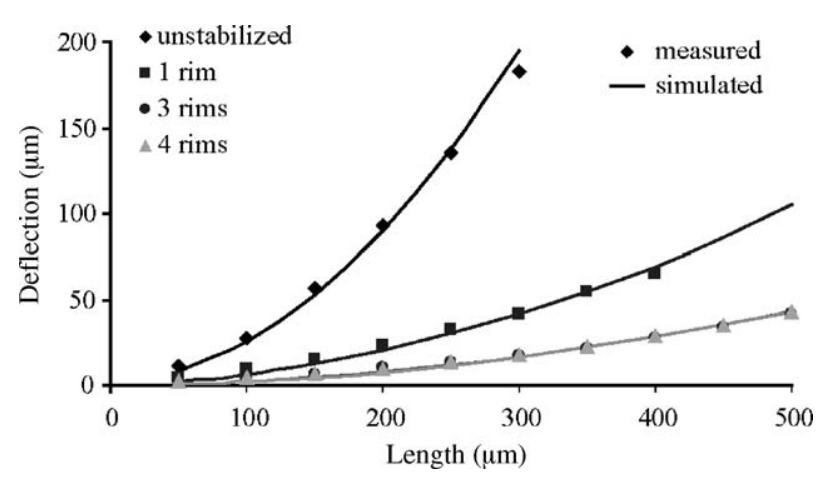

Fig. 8. Plot of simulation results (solid lines) vs. measurement data (dots) for corrugated cantilevers with a variable number of $1-\mu \mathrm{m}$ high rims.

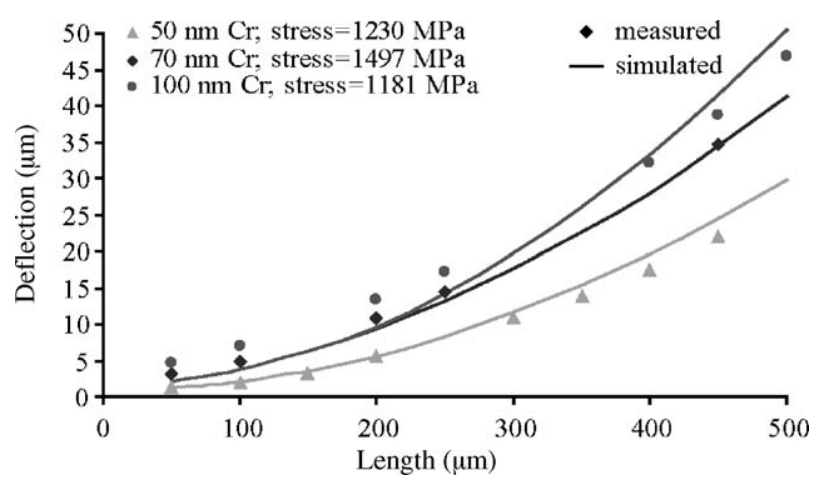

Fig. 9. Plot of simulation results (solid lines) vs. measurement data (dots) for cantilevers stabilized with one rim of $1 \mu \mathrm{m}$ in height for several thicknesses of the deposited Cr layer.

indicate a good correlation between simulation and experiments, positively validating the FEM modeling tool as a reliable means of prediction of stress-induced deformation.

\section{Conclusions}

Corrugated membranes were fabricated for use as stabilized stencil deposition membranes. The after-deposition deflections of unstabilized and corrugated (stabilized) membrane structures were determined experimentally and by FEM simulations. 
SEM and DFM images indicate an increased mechanical stability of the membranes and improved pattern definition with stencil lithography. The simulation and experimental results show that introducing stabilization structures in the membrane can significantly reduce (up to $96 \%$ ) the out-of-plane deformation of the membranes. Good agreement between experimental results and FEM simulations was obtained confirming the FEM tool as a reliable means for modeling the stressinduced deformation. Verified FEM models will be used as guidelines in future work to find optimal stabilization schemes for complex membranes. This will allow resistless nanostencil lithography on large surfaces with improved surface pattern definition.

\section{Acknowledgements}

We are pleased to acknowledge the EPFL Center of MicroNano-Technology (CMI) and its entire staff as well as our colleagues at the Microsystems Laboratory and the Computational Modeling Group at Tyndall National Institute for their very valuable discussions and help. This project is partially funded by the European Commission and OFES within the project $\mathrm{NaPa}$ (Contract no. NMP3-CT-2003-500120) and by the Swiss National Science Foundation project NANO-IC (Contract no. 200021101847).

\section{References}

[1] S.Y. Chou, P.R. Krauss, P.J. Renstrom, Nanoimprint lithography, J. Vac. Sci. Technol. B 14 (6) (1996) 4129-4133.

[2] S. Johnson, R. Burns, E.K. Kim, G. Schmid, M. Dicky, J. Meiring, S. Burns, N. Stacey, C.G. Wilson, D. Convey, Y. Wei, P. Fejes, K. Gehoski, D. Mancini, K. Nordquist, W.J. Dauksher, D.J. Resnick, Step and flash imprint lithography modeling and process development, J. Photopolym. Sci. Technol. 17 (2004) 417-419.

[3] B. Michel, A. Bernard, A. Bietsch, E. Delamarche, M. Giessler, D. Juncker, K. Kind, J.-P. Renault, H. Rothuizen, H. Schmid, P. SchmidtWinkel, R. Stutz, H. Wolf, Printing meets lithography: soft approaches to high-resolution patterning, IBM J. Res. Dev. 45 (5) (2001) 697719.

[4] A. Kumar, G.M. Whitesides, Features of gold having micrometer to centimeter dimensions can be formed through a combination of stamping with an elastomeric stamp and an alkanethiol "ink" followed by chemical etching, Appl. Phys. Lett. 63 (14) (1993) 2002-2004.

[5] R.D. Piner, J. Zhu, F. Xu, S. Hong, C.A. Mirkin, "Dip-pen" nanolithography, Science 283 (5402) (1999) 661-663.

[6] A. Meister, S. Jeney, M. Liley, T. Akiyama, U. Staufer, N.F. de Rooij, H. Heinzelmann, Nanoscale dispensing of liquids through cantilevered probes, Microelectron. Eng. 67-68 (2003) 644-650.

[7] G.M. Kim, M.A.F. van den Boogaart, J. Brugger, Fabrication and application of a full wafer size micro/nanostencil for multiple lengthscale surface patterning, Microelectron. Eng. 67-68 (2003) 609614.

[8] J. Koehler, M. Albrecht, C.R. Musil, E. Bucher, Direct growth of nanostructures by deposition through an $\mathrm{Si}_{3} \mathrm{~N}_{4}$ shadow mask, Physica E: Low-dimensional Syst. Nanostruct. 4 (3) (1999) 196-200.

[9] M.M. Deshmukh, D.C. Ralph, M. Thomas, J. Silcox, Nanofabrication using a stencil mask, Appl. Phys. Lett. 75 (11) (1999) 1631-1633.

[10] J. Brugger, J.W. Berenschot, S. Kuiper, W. Nijdam, B. Otter, M. Elwenspoek, Resistless patterning of sub-micron structures by evaporation through nanostencils, Microelectron. Eng. 53 (1-4) (2000) 403-405.
[11] M.A.F. van den Boogaart, G.M. Kim, R. Pellens, J.-P. van den Heuvel, J. Brugger, DUV-MEMS stencils for high-throughput resistless patterning of mesoscopic structures, J. Vac. Sci. Technol. B 22 (6) (2004) 3174-3177.

[12] M. Ohring, The Material Science of Thin Films, Academic Press, 1992.

[13] K.-S. Chen, X. Zhang, S.-Y. Lin, Intrinsic stress generation and relaxation of plasma-enhanced chemical vapor deposited oxide during deposition and subsequent thermal cycling, Thin Solid Films 434 (1-2) (2003) 190-202.

[14] A. Tarraf, J. Daleiden, S. Irmer, D. Prasai, H. Hillmer, Stress investigation of PECVD dielectric layers for advanced optical MEMS, J. Micromech. Microeng. 14 (3) (2004) 317-323.

[15] R. Hammer, Solving the "stencil" problem in vacuum deposition masks using rib-supported structures, J. Vac. Sci. Technol. 14 (5) (1977) $1208-1210$.

[16] M.A.F. van den Boogaart, L.M. Doeswijk, J. Brugger, Silicon supported membranes for improved large-area and high-density nanostencil lithography, JMEMS, submitted for publication.

[17] T. Ondarçuhu, L. Nicu, S. Cholet, C. Bergaud, S. Gerdes, C. Joachim, A metallic microcantilever electric contact probe array incorporated in an atomic force microscope, Rev. Sci. Instrum. 71 (5) (2000) 2087-2093.

[18] ConventorWare. Reference Guides and Tutorials.

[19] H. Baltes, O. Brand, G.K. Fedder, C. Hierold, J.G. Korvink, O. Tabata, CMOS-MEMS, Wiley/VHH, Weinhem, 2005.

[20] R. Whiting, M.A. Angadi, Young's modulus of thin films using a simplified vibrating reed method, Meas. Sci. Technol. 1 (7) (1990) 662

\section{Biographies}

Marc A.F. van den Boogaart received a BSc degree in precision engineering with a specialization in microengineering from the "Hogeschool van Utrecht", Utrecht, The Netherlands, in 2000. From 2000 to 2002, he worked on the development of thin-film microelectrode arrays, designed for interaction with cultured neurons at the University of Twente, Enschede, The Netherlands. $\mathrm{He}$ is currently working towards the $\mathrm{PhD}$ degree in microengineering on the development of micro- and nanostencils for stencil lithography at the Ecole Polytechnique Fédérale de Lausanne (EPFL), Lausanne, Switzerland.

Maryna Lishchynska received a MSc in applied mathematics from Lviv Polytechnic State University, Ukraine, in 1993, and was awarded a PhD in technical sciences from the Institute of Physics and Mechanics of the National Academy of Sciences of Ukraine in 2000. Dr. Lishchynska's PhD research was in the area of deformable solid mechanics (fatigue fracture mechanics). During 1993-2001, she worked as a research scientist on fracture mechanics problems for the Institute of Physics and Mechanics of the National Academy of Sciences of Ukraine and published a range of papers. Maryna joined the Tyndall National Institute (Cork, Ireland) in 2003 as a post-doctoral researcher in the Computational Modeling Group. Her current research interests include development of behavioral models of microsystems and application of FEM modeling tools to the solution of mechanical problems in MEMS structures.

Lianne M. Doeswijk received her MSc degree in applied physics with a specialization in heat transfer and fluid dynamics from the University of Twente (Enschede, The Netherlands) in 1997. She was awarded a PhD degree in 2002 from the University of Twente for her thesis: 'Pulsed Laser Deposition of Oxide Films on Silicon: Exploring their Passivating Qualities'. She is currently working on the development of stencil lithography at the Ecole Polytechnique Fédérale de Lausanne (EPFL), Lausanne, Switzerland.

James C. Greer received a Bachelor of Science in electrical engineering from the University of Texas at Austin, a Master of Science from Trinity College Dublin and the Doctor rerum naturalium (Dr. rer. nat.) from Albert-Ludwigs-Universität, Freiburg, Germany. He has worked for United Technologies Mostek, Texas Instruments and Hitachi Central Research Laboratory on topics in semiconductor device physics. Before moving to the Tyndall National Institute (previously named the NMRC, Ireland) in 1997, he taught and performed research on scientific computation at Trinity College Dublin. 
Jürgen Brugger received a $\mathrm{PhD}$ degree in 1995 from the University of Neuchatel, Switzerland, for a work on microfabricated tools for the atomic force microscope, which included a one-year period at the Hitachi Central Research Laboratories, Tokyo, Japan. He then joined the IBM Zurich Research Laboratory in Rüschlikon, Switzerland. From 1998 to 2001, he was directing the "NanoLink" Strategic Research Orientations at the MESA+ Research Institute, University of Twente, The Netherlands. In September
2001, he was appointed assistant professor "tenure track" at the EPFL. His main professional interests include the development of new tools for nanoscience and engineering techniques at the mesoscopic length-scale, in particular to develop methods for accessing the nanometer scale (top-down), and to combine them with self-assembly strategies (bottom-up), and to bridge life-science with solid-state devices at the sub-micrometer scale. 